\title{
Water as the solvent for the process of phenol hydroxylation over the Ti-MWW catalyst
}

\author{
Agnieszka Wróblewska
}

Received: 29 August 2012/Accepted: 29 October 2012/Published online: 20 November 2012

(C) The Author(s) 2012. This article is published with open access at Springerlink.com

\begin{abstract}
This work presents the studies on the process of phenol hydroxylation over the Ti-MWW catalyst. The medium of the reaction was water, whose amount was a sum of water introduced into the reactor with the $30 \mathrm{wt} \%$ hydrogen peroxide (oxidizing agent) and formed during the hydroxylation from the hydrogen peroxide (the first variant of the process) or water, which was the sum of water introduced additionally to the reaction mixture, water introduced with the oxidizing agent and formed during the reaction from the hydrogen peroxide (the second variant of the process). Studies have shown that in water solution, it is best to conduct phenol hydroxylation process at the temperature of $100{ }^{\circ} \mathrm{C}$, phenol/hydrogen peroxide molar ratio 1 , water content $62 \mathrm{wt} \%$, catalyst content $8 \mathrm{wt} \%$ and during the reaction time of $180 \mathrm{~min}$. Under these conditions, it is possible to achieve a selectivity of hydroquinone $43 \mathrm{~mol} \%$. A total conversion of phenol $28 \mathrm{~mol} \%$, and a selectivity of transformation to organic compounds in relation to phenol consumed $97 \mathrm{~mol} \%$.
\end{abstract}

Keywords Phenol hydroxylation - Water as a solvent - Ti-MWW catalyst

\section{Introduction}

Hydroquinone and pyrocatechol are very useful semi-products for the chemical industry. These compounds are used in medicine (contraceptives, urinary tract medicinal preparation, acne treating preparations, antidepressants, and anorexia medicinal preparation), in the cosmetics industry (skin bleaching creams, fragrance components), in food industry (flavors) and in the polymer industry (bearings, heatresistant cable insulations, films and plates for electrical circuit boards) [1, 2].

\footnotetext{
A. Wróblewska $(\bowtie)$

Institute of Organic Chemical Technology, West Pomeranian University of Technology, Szczecin, Pułaskiego 10, Pl 70-322 Szczecin, Poland

e-mail: Agnieszka.Wroblewska@zut.edu.pl
} 
On an industrial scale, the phenol hydroxylation process (installation by Enichem Company) is conducted in a liquid phase at the temperature of $100{ }^{\circ} \mathrm{C}$, when the molar ratio phenol/hydrogen peroxide is $3: 1$, the methanol concentration is $30 \mathrm{wt} \%$, and the TS- 1 content is $3 \mathrm{wt} \%$. The molar ratio of products (pyrocatechol/hydroquinone) is $1: 1$, the summary selectivity of the main products in relation to phenol consumed amounts to 90-94 mol\%, and the conversion of phenol is $25-30 \mathrm{~mol} \%$ [1, 3, 4]. A main disadvantage connected with the utilization of the TS- 1 catalyst is a low reproducibility of its synthesis (taking into account the content of titanium) and leaching of titanium from the structure during hydroxylation process. The same problem also appears in the case of the other titanium silicalite catalysts: TS-2 [5] or Ti-MCM-41 [6]. One of the newest titanium silicalite catalysts is the Ti-MWW catalyst [7]. Our preliminary studies indicate that the syntheses of Ti-MWW catalyst are more reproducible and the catalyst is characterized by greater stability [8]. Hence, it would be interesting to verify the applicability of Ti-MWW catalyst instead of TS- 1 catalyst in this process. Another disadvantage associated with the industrial process of phenol hydroxylation is the use of large amounts of methanol (solvent) in the process (30 wt \%). From both technological and environmental points of view, it would be beneficial to develop variants of this process carried out in the aqueous medium, wherein the amount of water used in the process is only a sum of water which come from solution of the oxidizing agenthydrogen peroxide (30 wt $\%$ aqueous solution of the compound is used in the process) and water which is formed in the process (the first variant of this process-hydroxylation of phenol without added external water) or the amount of water used in the process is a sum of water which is introduced additionally to the reaction mixture, water introduced with oxidizing agent and water formed in the process (the second variant of this process-hydroxylation of phenol with added external water). The aim of this study is to investigate the potential for a successful conduct of phenol hydroxylation process over Ti-MWW catalyst in water environment in these two variants. The results will be compared with our previous results obtained during phenol hydroxylation process over Ti-MWW catalyst, but in an acetonitrile medium.

\section{Experimental}

For the synthesis of the titanium-silicalite Ti-MWW catalyst, the following raw materials were applied: tetrabutyl $o$-titanate (TBOT, $\mathrm{Ti}(\mathrm{OBu})_{4}, 95 \%$, Fluka), hexamethyleneimine (HMI, $\mathrm{C}_{6} \mathrm{H}_{13} \mathrm{~N},>98 \%$, Merck), boric acid $\left(\mathrm{H}_{3} \mathrm{BO}_{3}, 99.5 \%\right.$, Chempur), and fumed silica (Cab-o-sil M5, Biesterfeld). The Ti-MWW catalyst was synthesized by the method of $\mathrm{Wu}$ et al. [9]. The full characteristic of this catalyst was presented in our previous article [8].

For the hydroxylation of phenol, the following raw materials were used: phenol (>99\%, Aldrich) and hydrogen peroxide (30 wt\% water solution, POCH Gliwice). Hydroxylation of phenol was carried out in a stainless steel autoclave equipped with a PTFE insert of $60 \mathrm{~cm}^{3}$ capacity. Raw materials were introduced into the reactor in the following order: catalyst, phenol, and hydrogen peroxide. The reactor was located in a shaker holder and immersed in an oil bath (the intensity of stirring $300 \mathrm{rpm}$ ). The bath temperature was controlled by a thermostat. After completing the reaction, the autoclave was cooled and weighed. 
The post-reaction mixtures were analyzed quantitatively using gas chromatography (the amounts of phenol, hydroquinone, pyrocatechol and $p$-benzoquinone). The chromatographic analyses were performed on a Focus apparatus equipped with a flame-ionization detector and using a capillary column Restek Carbowax $(30 \mathrm{~m} \times 0.53 \mathrm{~mm} \times 0.25 \mu \mathrm{m})$. The qualitative analyses were performed on a Hewlett Packard 6890 apparatus equipped with a mass detector HP 5973 and using a capillary column HP $5 \mathrm{MS}(30 \mathrm{~m} \times 0.25 \mathrm{~mm} \times 0.25 \mu \mathrm{m})$.

The product analyses allowed to calculate the main functions describing the process: the selectivity of hydroquinone $\left(\mathrm{S}_{\text {hydroquinone/phenol }}\right)$, the total conversion of phenol $\left(\mathrm{C}_{\text {phenol }}\right)$, and the selectivity of transformation to organic compounds in relation to phenol consumed $\left(\mathrm{S}_{\text {org. comp./phenol }}\right)$. These functions were calculated according the following formulas:

$$
\begin{aligned}
& \mathrm{S}_{\text {hydroquinone } / \text { phenol }} \\
& =\frac{\text { Amount of hydroquinone obtained }(\mathrm{mol})}{\text { Amount of phenol react to hydroquinone, pyrocatechnol and } p \text {-benzoquinone }(\mathrm{mol})} \\
& \times 100 \% \\
& \mathrm{C}_{\text {phenol }}=\frac{\text { Amount of phenol consumed }(\mathrm{mol})}{\text { Initial amount of phenol }} \times 100 \% \\
& \mathrm{~S}_{\text {org.comp./phenol }} \\
& =\frac{\text { Amount of organic compound obtained (hydroquinone and pyrocatechnol })(\mathrm{mol})}{\text { Amount of phenol consumed }(\mathrm{mol})} \\
& \quad \times 100 \%
\end{aligned}
$$

Very important functions describing the process of phenol hydroxylation are also the conversion of phenol to tars and the selectivity of transformation to tars in relation to phenol consumed. The first function can be calculated as the difference between the conversion of phenol (the total conversion of phenol $\mathrm{C}_{\text {phenol }}$ ) and the conversion of phenol to organic compounds (hydroquinone, pyrocatechol and $p$-benzoquinone). The conversion of phenol to organic compounds can be calculated as follows:

$$
\begin{aligned}
& \mathrm{C}_{\text {phenol to org. comp. }}=\frac{\text { Amount of hydroquinone, pyrocatechol and } p \text {-benzoquinone }(\mathrm{mol})}{\text { Initial amount of phenol }(\mathrm{mol})} \\
& \quad \times 100 \%
\end{aligned}
$$

The second function (the selectivity of transformation to tars in relation to phenol consumed) can be calculated as follows:

$$
\mathrm{S}_{\text {tars } / \text { phenol }}=100 \%-\mathrm{S}_{\text {org. comp. } / \text { phenol }}
$$

\section{Results and discussion}

The process of phenol hydroxylation can be described by the following formulas [10]:-

In the first step of the reaction, two main products are formed: hydroquinone and pyrocatechol which can undergo further oxidation to $o$ - and $p$-quinones. From these 
two quinones $o$-quinone is very easily converted to tars. Simultaneously, the formation of $m$-dihydroxybenzene (resorcinol) is not observed [10].

Even today, various aspects of the reaction mechanism for the process of phenol hydroxylation are not yet fully understood. In the literature, it is generally agreed for protic solvents (alcohols, water) that protic molecules coordinate to titanium (the active center of the catalyst) expending its coordination sphere to 5 or 6 . In this way, five-membered rings are formed, which are active intermediate complexes for catalyzed by titanium silicalite catalysts reactions. In protic solvents, the mechanism of phenol hydroxylation to hydroquinone can be presented as follows [11]:

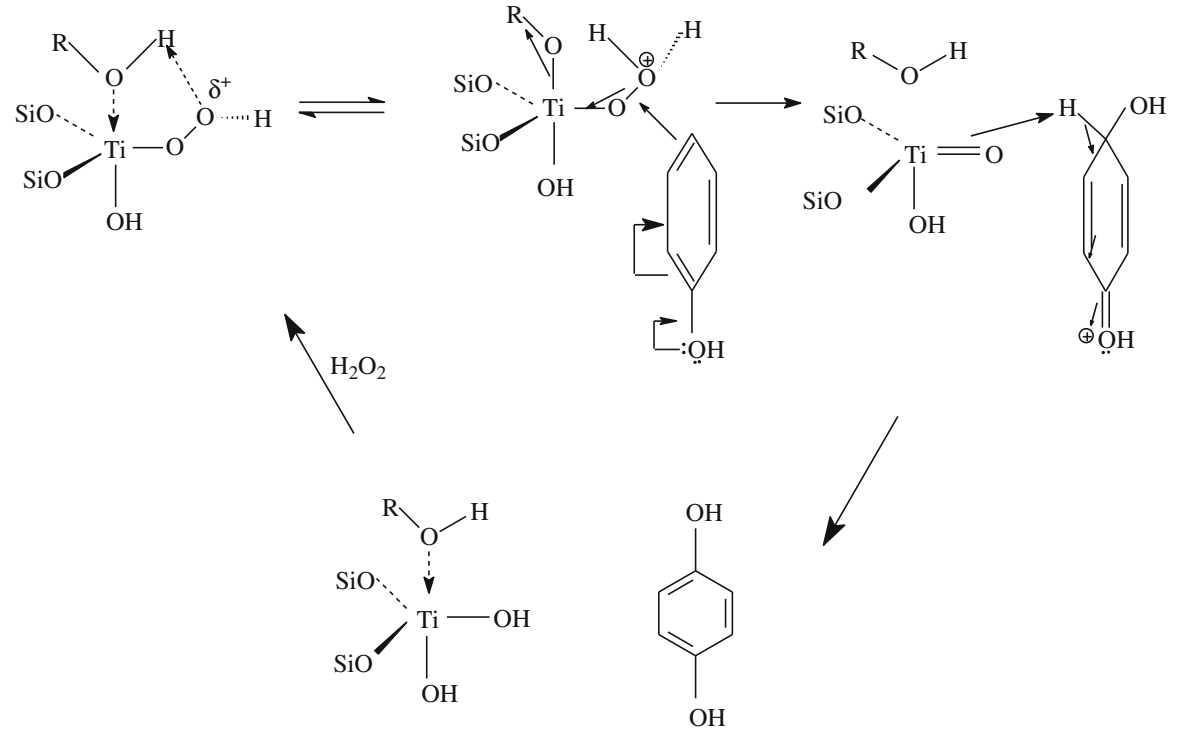

The formation of pyrocatechol in protic solvents can be explained as follows [11]:

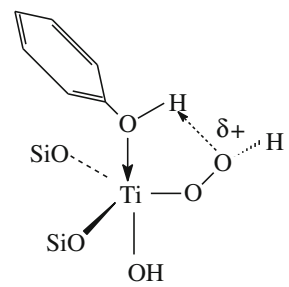

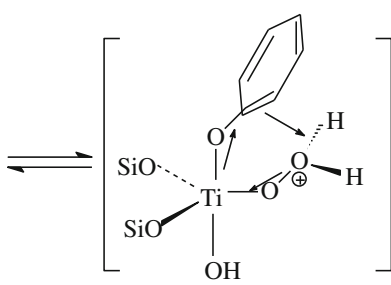

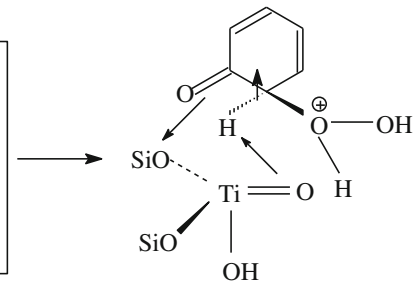<smiles>O=[18O]</smiles><smiles>[O-][Te](O)(O)(O)O</smiles><smiles>Oc1ccccc1O</smiles> 
or as follows [11]:

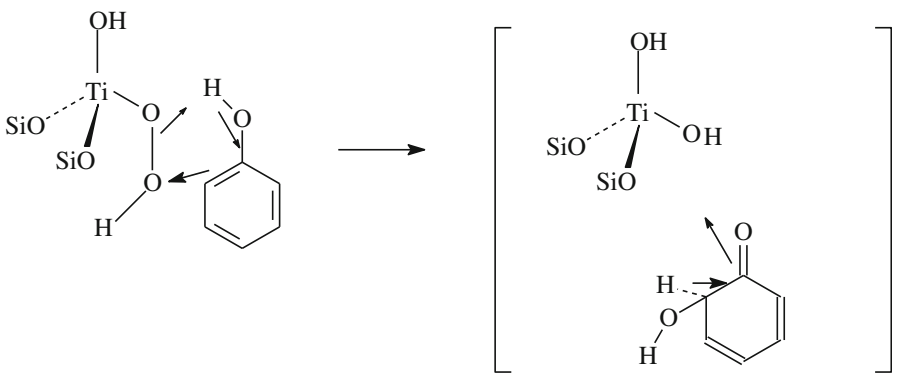
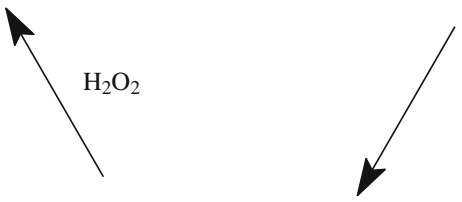<smiles>[O-][As]([O-])(O)(O)O</smiles><smiles>Oc1ccccc1O</smiles>

In the pores of the catalyst in the presence of protic solvents, the coordination of solvent molecules to the active center causes geometric strains thus the $p$-isomer (hydroquinone) is preferentially formed. The ratio of hydroquinone to pyrocatechol is always lower at the external surface than at the internal surface. It can be connected with the fact that at the external surface, the geometric strains are low and $o$-hydroxylation becomes more likely [11]. Simultaneously, on the external surface of the crystals, titanium is exposed to much higher concentrations of hydrogen peroxide. It leads to overoxidation and formation of tars what causes the poising of the external active centers, and this results in the formation of a higher amount of hydroquinone compared with pyrocatechol when the reaction time is lengthened.

Hydroxylation of phenol without added external water

The studies on the hydroxylation of phenol in the presence of water, which is the sum of water introduced into the reactor with $30 \mathrm{wt} \%$ hydrogen peroxide and formed during the hydroxylation was conducted at the following start parameters: the molar ratio phenol/hydrogen peroxide was $1: 1$, the content of Ti-MWW catalyst 
$10 \mathrm{wt} \%$ and the reaction time $120 \mathrm{~min}$. The concentration of water in the reaction mixture was about $37 \mathrm{wt} \%$, except studies on the influence of molar ratio of reactants, where for the molar ratio of phenol/hydrogen peroxide $=0.5$, water concentration was about $48 \mathrm{wt} \%$, for the molar ratio of phenol/hydrogen peroxide $=1$, water concentration was about $37 \mathrm{wt} \%$, for the molar ratio of phenol/ hydrogen peroxide $=2$, water concentration was about $25 \mathrm{wt} \%$ and for the molar ratio of phenol/hydrogen peroxide $=3$, water concentration was about $18 \mathrm{wt} \%$.

The influence of temperature on the course of phenol hydroxylation process is presented in Fig. 1.

Fig. 1 shows that the selectivity of hydroquinone is almost constant in the investigated range of temperatures (53-54 mol\%). So, the post-reaction mixture contains a slight molar excess of hydroquinone in relation to pyrocatechol (the molar ratio hydroquinone/pyrocatechol $=1.2$ ). It is in accordance with the mechanism presented above. According to this mechanism, the tars formed during the process of phenol hydroxylation, cause the poisoning of the active centers of titanium on the external surface of the catalyst, which leads to the increase in the amount of hydroquinone in relation to pyrocatechol. This phenomenon can be already observed at the relatively low temperatures and intensifies with an increase of the temperature of the hydroxylation process. It can be also observed taking into account the courses of the last two functions of the process: the total conversion of phenol and the selectivity of transformation to organic compounds in relation to phenol consumed. The total conversion of phenol rises during the increasing of the temperature of the process conducting from $17 \mathrm{~mol} \%\left(50{ }^{\circ} \mathrm{C}\right)$ to $61 \mathrm{~mol} \%\left(120{ }^{\circ} \mathrm{C}\right)$, and the second function decreases from 64 to $22 \mathrm{~mol} \%$. It means that higher temperatures accelerate the process of pyrocatechol oxidation to quinones and next to tars what is visible in the increase of the selectivity of transformation to tars up until $78 \mathrm{~mol} \%$ at the temperature of $120^{\circ} \mathrm{C}$.

At this stage of studies, the most beneficial temperature was taken as $100{ }^{\circ} \mathrm{C}$. At this temperature, relatively high values of the total conversion of phenol (42 mol\%)

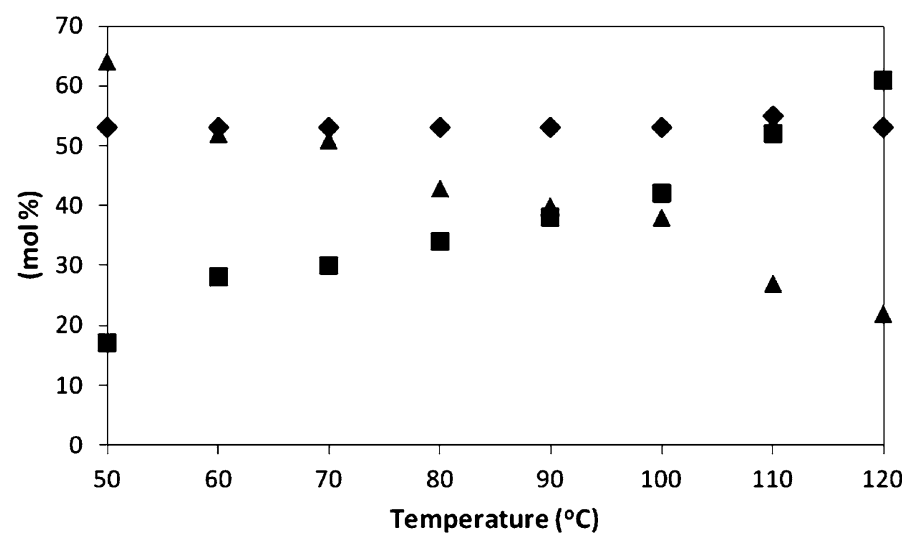

Fig. 1 The influence of temperature on the course of hydroxylation of phenol without added external water: filled diamond the selectivity of hydroquinone, filled square the total conversion of phenol, filled triangle the selectivity of transformation to organic compounds in relation to phenol consumed 
and selectivity of transformation to organic compounds in relation to phenol consumed are obtained (38 $\mathrm{mol} \%)$.

The studies on the influence of the molar ratio of phenol/hydrogen peroxide on the course of the phenol hydroxylation process are presented in Fig. 2. These studies were carried out at the temperature of $100{ }^{\circ} \mathrm{C}$, but the values of the next parameters were equal to the values of the start parameters.

Fig. 2 shows that a change in the molar ratio of reactants from 0.5 to 3 does not cause any changes in the selectivity of hydroquinone which amounts of 53-54 mol\%. However, with the increase of the molar ratio of reactants, the total conversion of phenol decreases, which is probably connected with a large excess of phenol in the reaction mixture in relation to the amount of oxidizing agent introduced into the reactor. With the increase of the molar ratio of phenol/hydrogen peroxide the selectivity of transformation to organic compounds in relation to phenol consumed slightly decrease from 36 to $26 \mathrm{~mol} \%$. Probably, at so high concentration of phenol in reaction mixture molecules of hydrogen peroxide have difficulties in the access to the pores, which causes that the process of the oxidation of the dihydroxybenzenes to quinones and next to tars at the external surface starts to dominate. A very essential influence on the course of the process is also played by the decrease the amount of water in the reaction mixture with the increase of molar ratio of reactants (from $48 \mathrm{wt} \%$ for the molar ratio phenol/hydrogen peroxide $0.5-18 \mathrm{wt} \%$ for the molar ratio phenol/hydrogen peroxide $=3$ ). It causes the amount of active complexes with titanium to decrease and the consequence of this is a decrease in the values of the conversion of phenol to organic compounds (for the molar ratio of reactants $=3$, the selectivity of tars amounts to $74 \mathrm{~mol} \%$ ).

At this stage of studies, the equimolecular molar ratio of phenol/hydrogen peroxide was taken as the most beneficial molar ratio of reactants. At this molar ratio, the total conversion of phenol and the selectivity of transformation to organic compounds in relation to phenol consumed have the highest values, 43 and $36 \mathrm{~mol} \%$.

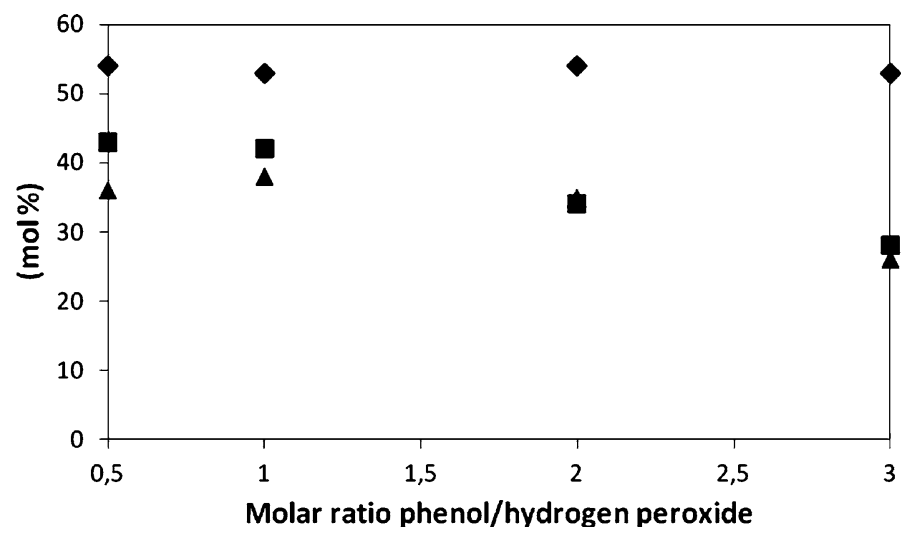

Fig. 2 The influence of the molar ratio phenol/hydrogen peroxide on the course of hydroxylation of phenol without added external water: filled diamond the selectivity of hydroquinone, filled square the total conversion of phenol, filled triangle the selectivity of transformation to organic compounds in relation to phenol consumed 


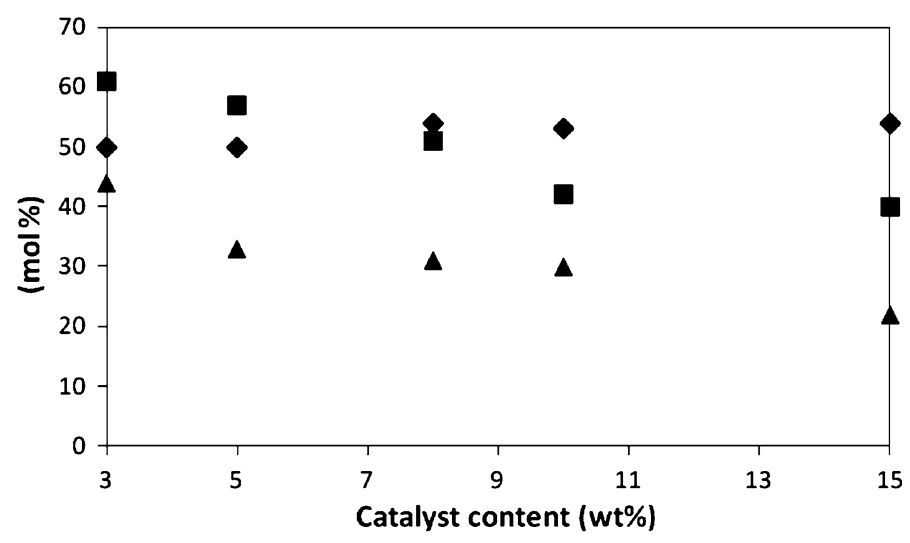

Fig. 3 The influence of the catalyst content on the course of hydroxylation of phenol without added external water: filled diamond the selectivity of hydroquinone, filled square the total conversion of phenol, filled triangle the selectivity of transformation to organic compounds in relation to phenol consumed

The studies on the influence of the catalyst content on the course of phenol hydroxylation process are presented in Fig. 3. These studies were carried out at the temperature of $100{ }^{\circ} \mathrm{C}$, at the equimolecular ratio of the reactants and for the reaction time of $120 \mathrm{~min}$.

Fig. 3 shows that with the increase of the content of the catalyst in the reaction mixture, the selectivity of hydroquinone changes slightly from $50 \mathrm{~mol} \%$ (the content of the catalyst amounts to $3 \mathrm{wt} \%$ ) to $54 \mathrm{~mol} \%$ (the content of the catalyst amounts to $15 \mathrm{wt} \%$ ). The total conversion of phenol as well as the selectivity of transformation to organic compounds decrease, the first function from 61 to $40 \mathrm{~mol} \%$, and the second from 44 to $22 \mathrm{~mol} \%$. The total conversion of phenol decreases probably because the catalyst is also active in an ineffective decomposition of hydrogen peroxide to water and oxygen and while an increase in the content of the catalyst intensifies this ineffective decomposition. The second reason of the decreasing total conversion of phenol can be connected with the fact that hydrogen peroxide takes part not only in the hydroxylation of phenol, but also in the oxidation of dihydroxybenzenes to quinones and next to tars (mainly pyrocatechol). This causes the increase in the selectivity of transformation to tars in relation to phenol consumed (the content of the catalyst $3 \mathrm{wt} \%$ - the selectivity to tars $56 \mathrm{~mol} \%$, the content of the catalyst $15 \mathrm{wt} \%$ - the selectivity to tars $78 \mathrm{~mol} \%$ ). The decrease in the selectivity values of transformation to organic compounds confirms this conclusion. Simultaneously, at low catalyst content (3 and $5 \mathrm{wt} \%$ ) the molar ratio phenol/hydrogen peroxide amounts to 1 , and next increase to 1.2 , which also confirms the fact that the decrease in amount of pyrocatechol takes place as a result of its oxidation to tars.

At this stage of studies, the content of $3 \mathrm{wt} \%$ was taken as the most beneficial catalyst content. At this catalyst content, the total conversion of phenol and selectivity of transformation to organic compounds in relation to phenol consumed have the highest values, 61 and $44 \mathrm{~mol} \%$. 
The studies on the influence of the reaction time on the course of phenol hydroxylation process are presented in Fig. 4. These studies were carried out at the temperature of $100{ }^{\circ} \mathrm{C}$, at the equimolecular ratio of the reactants, and at the catalyst content of $3 \mathrm{wt} \%$.

Fig. 4 shows that the prolongation of the reaction time from 15 to 180 min does not influence the selectivity of hydroquinone, which amounts to $50 \mathrm{~mol} \%$. On the other hand, the prolongation of the reaction time causes a slight increase in the total conversion of phenol from 56 to $67 \mathrm{~mol} \%$, at a simultaneous decrease in the selectivity of transformation to organic compounds in relation to phenol consumed from 45 to $31 \mathrm{~mol} \%$. The decrease in the values of the last function of the process is not high but it shows that the amount of tars in the reaction mixture rises for longer reaction times (reaction time $180 \mathrm{~min}$ - selectivity to tars $69 \mathrm{~mol} \%$ ).

At this stage of studies, the time of 120 min was taken as the most beneficial reaction time. For this reaction time, the total conversion of phenol and the selectivity of transformation to organic compounds in relation to phenol consumed have relatively high values, 61 and $44 \mathrm{~mol} \%$. Moreover, hydroquinone and pyrocatechol are obtained in equimolecular amounts.

Hydroxylation of phenol with added external water

The studies on the hydroxylation of phenol in the presence of water, which is the sum of water introduced additionally to the reaction mixture, water introduced with oxidizing agent and formed during the hydroxylation was conducted at the following start parameters: the molar ratio phenol/hydrogen peroxide $1: 1$, the content of water in the reaction mixture about $62 \mathrm{wt} \%$ (about $1.6 \mathrm{~g}$ of water was additionally introduced to the reaction mixture), the content of Ti-MWW catalyst $10 \mathrm{wt} \%$ and the reaction time of $180 \mathrm{~min}$. The content of water in the reaction mixture during the studies on the influence of the molar ratio of reactants was about

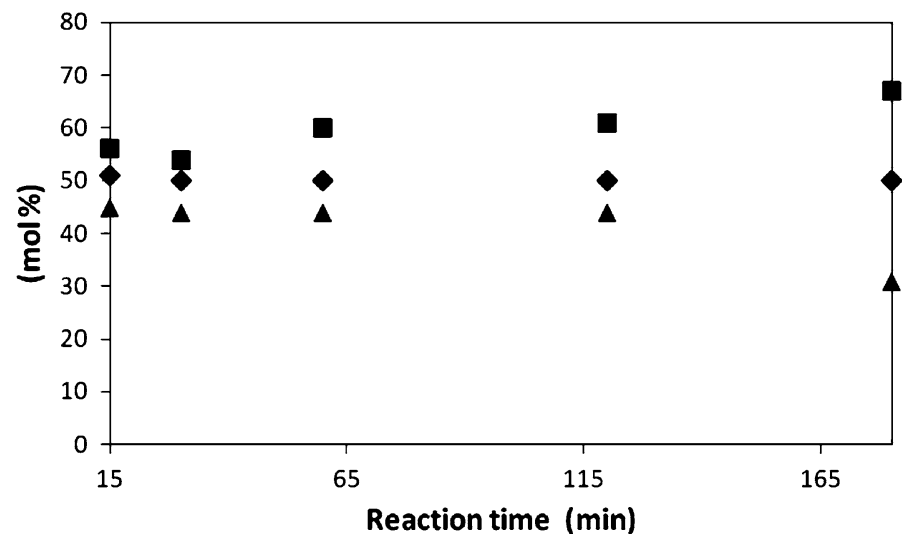

Fig. 4 The influence of the reaction time on the course of hydroxylation of phenol without added external water: filled diamond the selectivity of hydroquinone, filled square the total conversion of phenol, filled triangle the selectivity of transformation to organic compounds in relation to phenol consumed 
$69 \mathrm{wt} \%$ for the molar ratio of phenol/hydrogen peroxide $=0.5$, about $62 \mathrm{wt} \%$ for the molar ratio of phenol/hydrogen peroxide $=1$, about $55 \mathrm{wt} \%$ for the molar ratio of phenol/hydrogen peroxide $=2$, and about $52 \mathrm{wt} \%$ for the molar ratio of phenol/ hydrogen peroxide $=3$.

The influence of temperature on the course of phenol hydroxylation process was presented in Fig. 5.

Fig. 5 shows that with the increasing of the temperature of the process from 50 to $150{ }^{\circ} \mathrm{C}$, the selectivity of hydroquinone is practically constant and amounts to $41-42 \mathrm{~mol} \%$. Thus, a slight excess of pyrocatechol in relation to hydroquinone is obtained (the molar ratio of hydroquinone/pyrocatechol is 0.84 ). It shows that in the presence of higher concentrations of water, molecules of hydrogen peroxide have difficulties in accessing the internal surface of the catalyst and the hydroxylation mainly takes place on the external surface of the catalyst. It causes that larger amounts of pyrocatechol are obtained in relation to hydroquinone. The results obtained in the second variant of the process are different from the results obtained at the same stage, but in the first variant of the process hydroquinone slightly predominated in the product. Fig. 5 also shows that with the increase of the temperature of the process, the total conversion of phenol rises from $10 \mathrm{~mol} \%$ $\left(50{ }^{\circ} \mathrm{C}\right)$ to $43 \mathrm{~mol} \%\left(150{ }^{\circ} \mathrm{C}\right)$, but the selectivity of transformation to organic compounds in relation to phenol consumed decreases from 100 to $57 \mathrm{~mol} \%$. Moreover, there is visible the range of temperatures $\left(50-100{ }^{\circ} \mathrm{C}\right)$, in which tars are not formed during the process. This phenomenon was not observed in the first variant of the process performing.

At this stage of studies, $100{ }^{\circ} \mathrm{C}$ was taken as the most beneficial temperature. At this temperature, the very high value of the selectivity of transformation to organic compounds in relation to phenol consumed $(95 \mathrm{~mol} \%)$ can be obtained at an average value of the total conversion of phenol $(28 \mathrm{~mol} \%)$.

The studies on the influence of the molar ratio of phenol/hydrogen peroxide on the course of phenol hydroxylation process are presented in Fig. 6. These studies

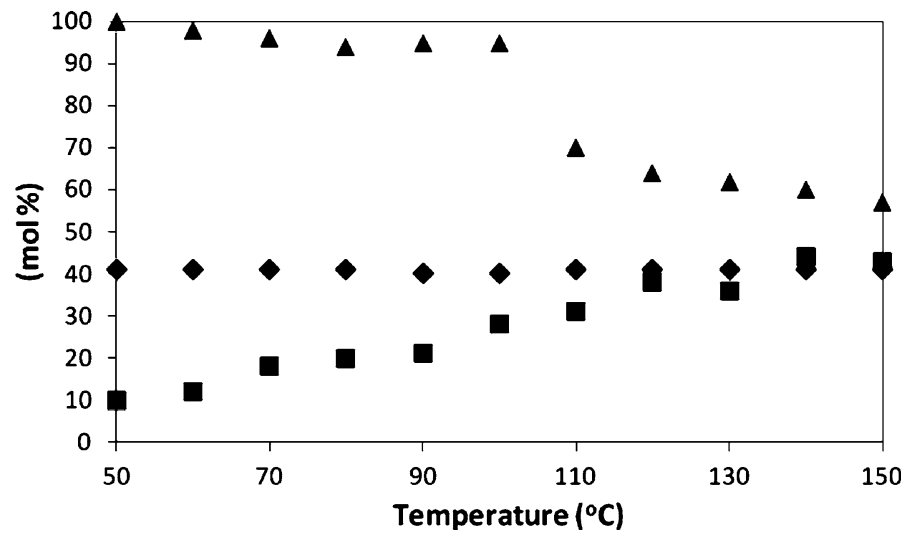

Fig. 5 The influence of temperature on the course of hydroxylation of phenol with added external water: filled diamond the selectivity of hydroquinone, filled square the total conversion of phenol, filled triangle the selectivity of transformation to organic compounds in relation to phenol consumed 
were carried out at the temperature of $100{ }^{\circ} \mathrm{C}$, but the values of the next parameters were equal to the values of the start parameters.

Fig. 6 shows that the change in the molar ratio of reactants does not influence on the selectivity of hydroquinone which amounts about 40-41 mol\% (a slight excess of pyrocatechol in the post-reaction mixture). The total conversion of phenol decreases during the increasing of the molar ratio of reactants from $68 \mathrm{~mol} \%$ (the molar ratio of reactants $=0.5$ ) to $22 \mathrm{~mol} \%$ (the molar ratio of reactants $=3$ ). For the molar ratio of reactants of 0.5 , despite the high total conversion of phenol, a low selectivity of transformation to organic compounds in relation to phenol consumed is obtained (45 mol\%) whereas at the equimolecular ratio of reactants the total conversion of phenol is much more lower $(28 \mathrm{~mol} \%)$, but the selectivity of transformation to organic compounds is very high and amounts to $95 \mathrm{~mol} \%$. It is because the fact that the oxidation of the obtained dihydroxybenzenes to tars takes place easily at two times excess of hydrogen peroxide. At the equimolecular ratio of reactants, this process is hindered. The increase in the amount of phenol in the reaction mixture (the molar ratio of reactants 2 and 3) causes a slight decrease in the total conversion of phenol (from 28 to $22 \mathrm{~mol} \%$ ) and also in the selectivity of transformation to organic compounds (from 44 to $29 \mathrm{~mol} \%$ ). Probably, at so high phenol concentration, the molecules of hydrogen peroxide have difficulties in accessing the internal surface. However, on the external surface, the hydrogen peroxide molecules participate in a greater amount in the oxidation of dihydroxybenzenes than in hydroxylation of phenol.

At this stage of studies, the equimolecular molar ratio of phenol/hydrogen peroxide was taken as the most beneficial molar ratio of reactants.

The studies on the influence of water content on the course of phenol hydroxylation process are presented in Fig. 7 (the content of water was changed from 36 to $89 \mathrm{~mol} \%$ ). These studies were carried out at the temperature of $100{ }^{\circ} \mathrm{C}$ at

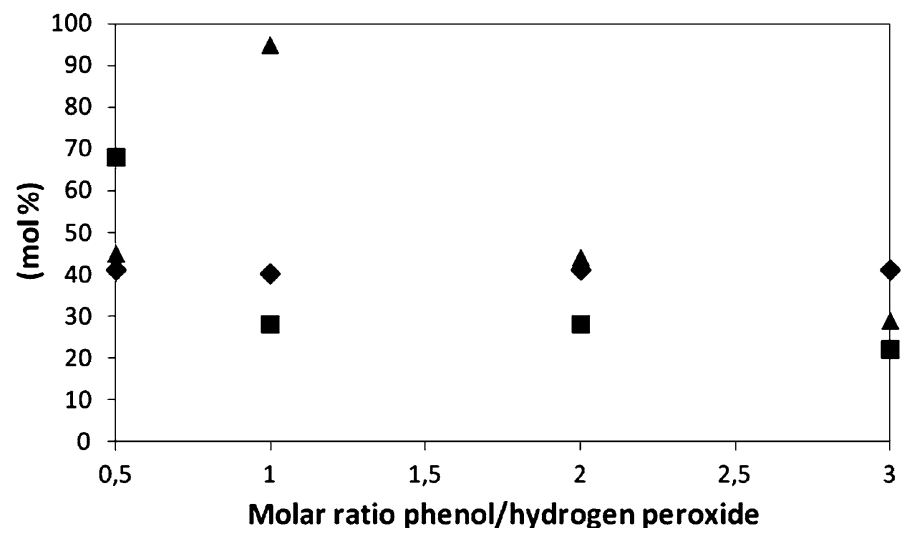

Fig. 6 The influence of the molar ratio of phenol/hydrogen peroxide on the course of hydroxylation of phenol with added external water: filled diamond the selectivity of hydroquinone, filled square the total conversion of phenol, filled triangle the selectivity of transformation to organic compounds in relation to phenol consumed 
the equimolecular ratio of the reactants, but the values of the next parameters were equal to the values of the starting parameters.

The studies on the influence of water content show that the increase in the amount of water from 36 to $89 \mathrm{wt} \%$ does not cause any changes in the selectivity of hydroquinone $(41 \mathrm{~mol} \%)$. Similarly, the increase in water content from 36 to $75 \mathrm{wt} \%$ does not change the total conversion of phenol which amounts to $26-28 \mathrm{~mol} \%$. Only for the water content of $89 \mathrm{wt} \%$, this conversion decreases to $18 \mathrm{~mol} \%$, which can be caused by a dilution of the reaction medium, and, consequently, by the difficulties in performing the hydroxylation. The selectivity of transformation to organic compounds at the water content amounting to 36 and $50 \mathrm{wt} \%$ reaches 40 and $57 \mathrm{~mol} \%$. At higher water content, a considerable inhibition of the process of dihydroxybenzene oxidation (the selectivity of organic compounds amounts to $95 \mathrm{~mol} \%$ ) is observed.

At this stage of studies, the content of $62 \mathrm{wt} \%$ was taken as the most beneficial water content. It allows to obtain $95 \mathrm{~mol} \%$ selectivity of transformation to organic compounds at the total phenol conversion amounting to $28 \mathrm{~mol} \%$.

The studies on the influence of the catalyst content on the course of phenol hydroxylation process are presented in Fig. 8. These studies were carried out at the temperature of $100{ }^{\circ} \mathrm{C}$, at the equimolecular ratio of the reactants, at the water content $62 \mathrm{wt} \%$ and for the reaction time $180 \mathrm{~min}$.

Fig. 8 shows that a change in the content of the catalyst does not have a significant influence on the selectivity of hydroquinone which amounts to 40-43 mol\%. The second function, the total conversion of phenol rises with the increase of the catalyst content from $28 \mathrm{~mol} \%$ ( $3 \mathrm{wt} \%$ of the catalyst) to $35 \mathrm{wt} \%$ (18 wt $\%$ of the catalyst). The selectivity of organic compounds has high values: $97-95 \mathrm{~mol} \%$ (3-10 wt $\%$ of the catalyst), $85 \mathrm{~mol} \%$ (15 wt $\%$ of the catalyst) and $76 \mathrm{~mol} \%$ (18 wt\% of the catalyst). In comparison with the first variant of this process, lower values of the total phenol conversion (almost two times) are obtained. This can be connected with a high dilution of the reaction mixture by

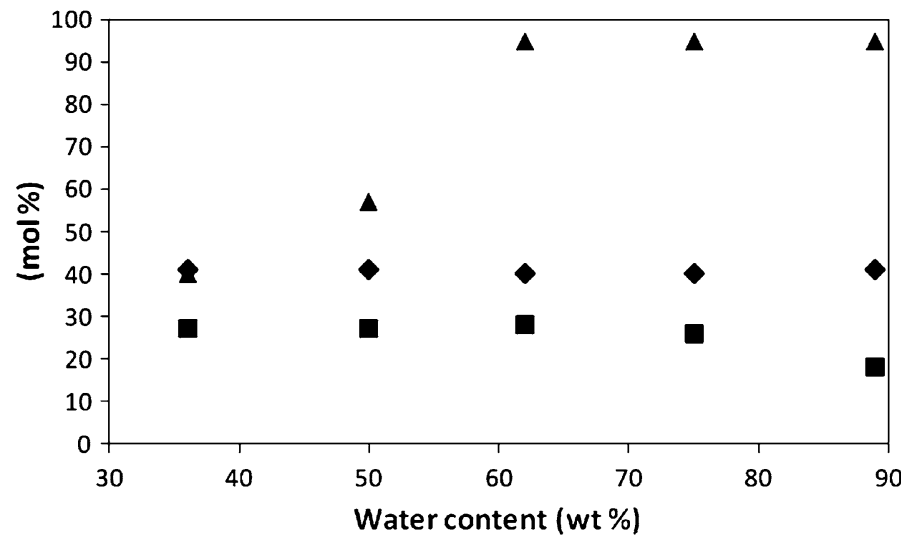

Fig. 7 The influence of water content on the course of hydroxylation of phenol with added external water: filled diamond the selectivity of hydroquinone, filled square the total conversion of phenol, filled triangle the selectivity of transformation to organic compounds in relation to phenol consumed 


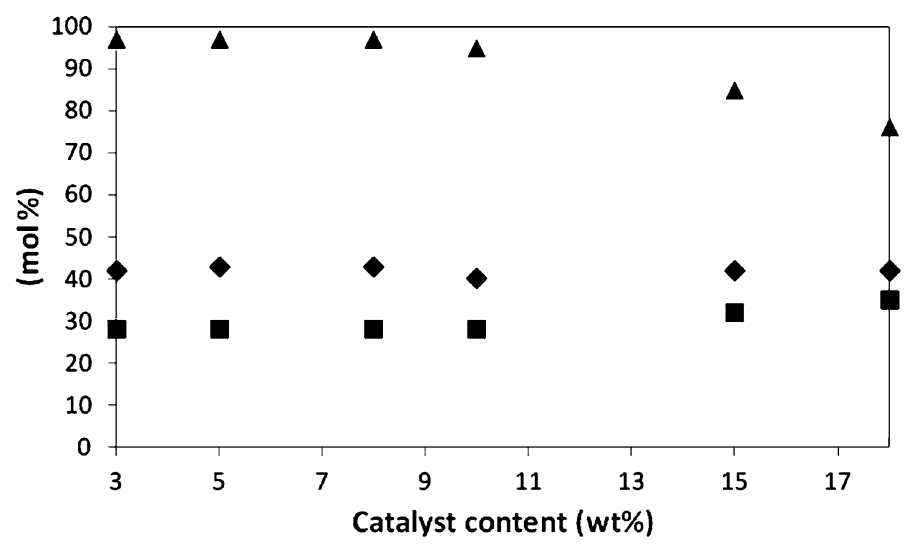

Fig. 8 The influence of the catalyst content on the course of hydroxylation of phenol with added external water: filled diamond the selectivity of hydroquinone, filled square the total conversion of phenol, filled triangle the selectivity of transformation to organic compounds in relation to phenol consumed

water. Simultaneously, in the comparison with the results obtained in the first variant of the process, as a result of higher water content utilization, it is possible to obtained high values of the selectivity to organic compounds (95-97 mol\%) at the catalyst content of 3-10 wt\% in the second variant of the process. At higher catalyst content, the amount of tars in the product is also rather small- $15 \mathrm{~mol} \%$ (15 wt $\%$ of the catalyst). In the first variant, he selectivity to tars was $78 \mathrm{~mol} \%$ for this catalyst content.

At this stage of studies, the content of $8 \mathrm{wt} \%$ was taken as the most beneficial catalyst content. It allows to obtain $97 \mathrm{~mol} \%$ selectivity of transformation to organic compounds at the total phenol conversion amounting to $28 \mathrm{~mol} \%$.

The studies on the influence of the reaction time on the course of phenol hydroxylation process are presented in Fig. 9. These studies were carried out at the

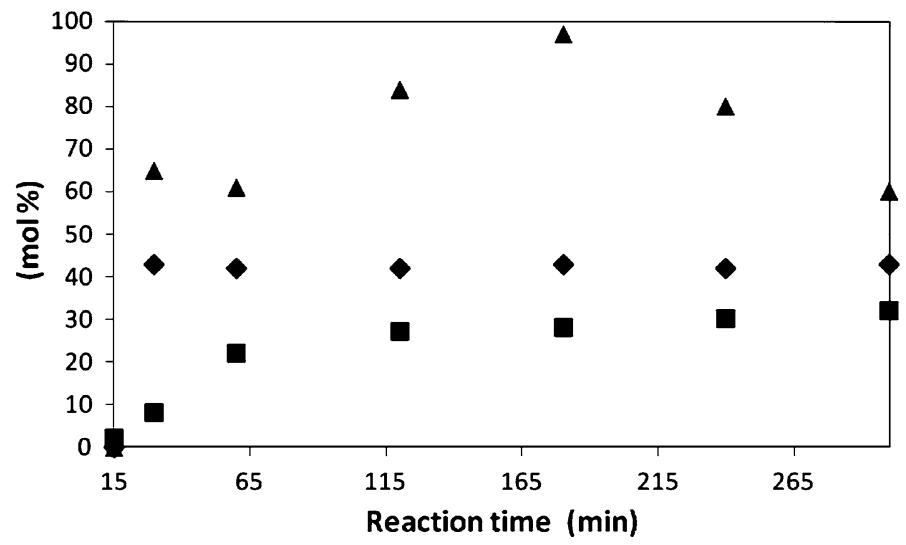

Fig. 9 The influence of the reaction time on the course of hydroxylation of phenol with added external water: filled diamond the selectivity of hydroquinone, filled square the total conversion of phenol, filled triangle the selectivity of transformation to organic compounds in relation to phenol consumed 
temperature of $100{ }^{\circ} \mathrm{C}$ at the equimolecular ratio of the reactants, water content of $62 \mathrm{wt} \%$ and the catalyst content of $8 \mathrm{wt} \%$.

Fig. 9 shows that the prolongation of the reaction time does not influence the selectivity of hydroquinone but has an essential influence on the next two functions of the process. The total conversion of phenol rises with the prolongation of the reaction time from $2 \mathrm{~mol} \%(15 \mathrm{~min})$ to $27-28 \mathrm{~mol} \%$ (120 $\mathrm{min})$. Longer reaction times do not cause any essential changes in values of this function (28-32 mol\%). The selectivity to organic compounds rises to $97 \mathrm{~mol} \%$ (180 $\mathrm{min})$ and next decreases to $60 \mathrm{~mol} \%$ (300 $\mathrm{min}$ ). It shows that for longer reaction times, the process of phenol hydroxylation will be inhibited and a great amount of dihydroxybenzenes will be oxidized to tars.

At this stage of studies, the time of 180 min was taken as the most beneficial reaction time.

\section{Conclusions}

The comparison of two variants of the process of phenol hydroxylation checked in this work shows that the most beneficial parameters established for these two variants are similar only in case of the temperature $\left(100{ }^{\circ} \mathrm{C}\right)$ and the molar ratio of reactants (the molar ratio phenol/hydrogen peroxide $=1$ ). The conducting of the process according to the first variant needs lower catalyst content- 3 wt\% (in the second variant the catalyst content amounts to $8 \mathrm{wt} \%$ ) and a shorter reaction time of $120 \mathrm{~min}$ (in the second variant the most beneficial reaction time amounts to $180 \mathrm{~min}$ ). The introduction of the additional amounts of water into the reaction mixture (in the first variant of the process water content was $37 \mathrm{wt} \%$ and in the second it was $62 \mathrm{wt} \%$ ) caused a slight decrease in the molar ratio of hydroquinone/ pyrocatechol from 1 to 0.8 . This shows that molecules of hydrogen peroxide had difficulties in accessing the active centers on the internal surface of the catalyst and the hydroxylation underwent easier on the external surface of the catalyst. Simultaneously, the introduction of the additional amounts of water into the reaction mixture caused the inhibition of the process of overoxidation (oxidation of the obtained dihydroxybenzenes to tars), which is very beneficial from the technological point of view. In the second variant of the process, the selectivity of tars amounted to $3 \mathrm{~mol} \%$, whereas in the first variant, it amounts to $56 \mathrm{~mol} \%$. Moreover, the use of the additional amount of water caused A decrease in the conversion of phenol from $61 \mathrm{~mol} \%$ (the first variant of the process) to $28 \mathrm{~mol} \%$ (the second variant of the process). However, it is beneficial from the technological point of view because phenol can be recycled and used again. The bigger problem is the formation large amounts of tars during the process, which is connected with ineffective conversion of phenol and with the loss of the catalyst activity (the regeneration of the catalyst is expensive and time-consuming). Taking into account the problems shown above, conducting the second variant of the process hydroxylation is better than the first.

The comparison of the results obtained in this work with our previous research carried out in acetonitrile medium [12] shows that the conducting the process of phenol hydroxylation in water medium is more beneficial. It needs a lower 
temperature of $100{ }^{\circ} \mathrm{C}$ (for the process in acetonitrile, the most beneficial temperature was $147{ }^{\circ} \mathrm{C}$ ), a lower catalyst content of $8 \mathrm{wt} \%$ (in the process in acetonitrile, the most beneficial catalyst content was 10.3-10.5 wt\%) and shorter reaction time $180 \mathrm{~min}$ (in the process in acetonitrile, the most beneficial reaction time was 221-236 $\mathrm{min}$ ). The content of water (62 wt\%) in the reaction medium is higher than in acetonitrile (21-24 wt\%), but it allows to obtain better values of the main function of the process (in acetonitrile, the selectivity of hydroquinone was $47 \mathrm{~mol} \%$, the conversion of phenol $76 \mathrm{~mol} \%$, and the selectivity to tars in relation to phenol consumed was $50 \mathrm{~mol} \%$ ).

The phenol hydroxylation method over Ti-MWW catalyst in aqueous medium, which was presented in this article, seems to be a preferred alternative to conventional solutions. It is more environmentally friendly and cost-effective, taking into account consumption of raw materials and energy.

Open Access This article is distributed under the terms of the Creative Commons Attribution License which permits any use, distribution, and reproduction in any medium, provided the original author(s) and the source are credited.

\section{References}

1. Wróblewska A, Milchert E (2002) Hydroksylowanie fenolu nadtlenkiem wodoru na katalizatorach tytanowo-silikalitowych. Przem Chem 81(8):515-518

2. Yu J, Yang P, Yang Y, Wu T, Paraquette JR (2006) Hydroxylation of phenol with hydrogen peroxide over tungstovanadophospates with Dawson structure. Catal Commun 7:153-156

3. Thangaraj A, Kumar R, Ratnasamy P (1991) Catalytic properties of crystalline titanium silicalite, Hydroxylation of phenol with hydrogen peroxide over TS-1 zeolites. J Catal 131:294-297

4. Tuel A, Moussa-Khouzzami S, Ben-Taarit Y, Naccache C (1991) Hydroxylation of phenol over TS1: surface and solvent effects. J Mol Catal 68:45-52

5. Tuel A, Ben-Taarit Y (1993) Comparison between TS-1 and TS-2 in the hydroxylation of phenol with hydrogen peroxide. Appl Catal A Gen 102:69-77

6. Lee GD, Kim SG, Hong HH (2007) Photocatalytic hydroxylation of phenol over Ti-containing zeolites (TS-1, Ti-MCM-41). Solid State Phenom 124-126:1793-1796

7. Fajdek A, Wróblewska A, Milchert E (2011) Selective liquid-phase oxidation of allyl alcohol to glycidol over MWW type titanosilicalite. Reac Kinet Mech Cat 103:451-462

8. Wróblewska A, Fajdek A, Milchert E, Grzmil B (2010) The Ti-MWW catalyst-its characteristic and catalytic properties in the epoxidation of allyl alcohol by hydrogen peroxide. Pol $\mathrm{J}$ Chem Technol 1(12):29-34

9. Wu P, Tatsumi T, Komatsu T, Yashima T (2001) A novel titanosilicate with MWW structure. I. Hydrothermal synthesis, elimination of extraframework titanium, and characterizations. J Phys Chem B 105(15):2897-2905

10. Reddy JS, Sivasanker S, Ratnasamy P (1992) Hydroxylation of phenol over TS-2, a titanium silicate molecular sieve. J Mol Catal 71:373-381

11. Wilkenhoner U, Langhendries G, van Laar F, Baron GV, Gammon DW, Jacobs PA, van Steen E (2001) Influence of pore and crystal size of crystalline titanosilicates on phenol hydroxylation in different solvents. J Catal 203:201-212

12. Wróblewska A (2012) Optimization of a Ti-MWW catalysed phenol hydroxylation process. J Adv Oxid Technol 15(2):418-428 